\title{
Hamiltonian systems with orbits covering densely submanifolds of small codimension
}

\author{
Ernest Fontich \\ Departament de Matemàtica Aplicada i Anàlisi \\ Universitat de Barcelona \\ Gran Via, 585, 08007 Barcelona, Spain \\ Pau Martín \\ Departament de Matemàtica Aplicada i Telemàtica \\ Universitat Politècnica de Catalunya \\ Ed-C3, Jordi Girona, 1-3, 08034 Barcelona Spain
}

July 21,2000

\begin{abstract}
The existence of a transition chain in a Hamiltonian system leads to the existence of orbits shadowing it, if some lambda lemma can be applied. This fact has been used to prove the existence of diffusion in perturbations of integrable a priori stable systems. We prove that, under suitable conditions there are orbits which cover densely codimension two submanifolds of the $(2 n-1)$-dimensional energy level. We also provide an example where the computations to verify the sufficient conditions for the appearance of such phenomenon can be performed. We comment on Arnol'd example, which is covered by this set of hypotheses.
\end{abstract}

AMS Subject Classification (2000): 37J40, 34C37, 70H05.

Keywords: Hamiltonian systems, invariant tori, Arnold diffusion.

\section{Introduction}

The notions of obstruction set, transition torus and transition chain were introduced by Arnol'd in his celebrated paper [1], where they were used to prove the existence 
of diffusion in a particular perturbation of an integrable Hamiltonian system. We briefly recall these notions: in some space $X$ we say that a set $\Omega \subset X$ is an obstruction set of $M$ at a point $x \in M$ if for every manifold $N$ which intersects transversely $M$ at $x$ in $X$ we have that $N \cap \Omega \neq \emptyset$. In applications to Hamiltonian systems, the obstruction set $\Omega$ is often taken as an invariant manifold intersecting transversely the stable manifold of a partially hyperbolic torus (see definition in Section 2), while $M$ is the unstable manifold of the torus. Let $H$ be a Hamiltonian and let $\phi_{t}$ be the flow generated by its associated vector field. We say that an invariant torus of $H$ is a transition torus if it is a partially hyperbolic torus such that for any neighborhood $U$ of any point of its stable manifold, $\left\{\phi_{t}(U) ; t \geq 0\right\}$ is an obstruction set of its unstable manifold at any point of it.

A transition chain is a (possibly infinite) sequence of transition tori such that the unstable manifold of each torus intersects transversally the stable manifold of the following one.

In the usual way to study Arnol'd diffusion, some transition chain is constructed. For that some tori are selected and then a suitable lambda lemma is provided to ensure that the tori are indeed transition tori $[4,5,17,11]$.

In [1], Assertion B states that the tori considered in the Arnol'd example are indeed transition tori. Other remarkable lambda lemma can be found in $[6,16]$.

In this paper we shall use the lambda lemma in [12], since it applies to general tori.

Then to find initial conditions for orbits shadowing the prescribed transition chains, we use simple topological arguments as it is indicated in Arnold-Avez [2, Lemma 23.8]. In this way the set of initial conditions is found in the intersection of some open sets. If we have a finite sequence of tori, then this set is open. However, if we have an infinite sequence, we can give an argument to prove that the set is non-void.

These arguments are also used in $[3,7]$ to find orbits with unbounded energy in a class of time dependent analytic Hamiltonian systems.

Transition chains connecting two neighborhoods of the phase space, if they can be found, are far from being uniquely determined. Since the next torus in the sequence is chosen in order that the corresponding invariant manifolds intersect transversally, any torus verifying this hypothesis is acceptable. In particular, from a given torus, it is possible to move in many directions. In this way, the orbits which shadow the chain may look like moving randomly.

The first part of the paper is devoted to study properties of partially hyperbolic tori in Hamiltonian systems. A simple normal form around a torus is presented. It this form, the results in [12] can be applied to get the existence of invariant manifolds associated to the hyperbolic directions of the torus. This normal form also permits to apply the lambda lemma in [12] to prove that the torus (and any $C^{1}$-close torus) 
has the obstruction property.

The main result of the present paper puts in an abstract context a set of sufficient conditions to find orbits which cover densely invariant submanifolds. With the above mentioned techniques, we will construct orbits which shadow transition chains with an infinite number of tori. This construction is quite general and can be applied in several situations. In our case, we have supplied enough hypotheses to find orbits which cover densely invariant manifolds (of codimension 3 ) foliated with invariant tori, that is, although on the manifolds the dynamics is confined on each torus, there is an actual orbit shadowing them and thus providing transport. Then an example which satisfies those conditions is presented. More concretely, we show that there is an orbit which shadows a whole resonance surface (for a fixed energy level). The same argument can be applied to those Hamiltonian systems in [11] to obtain orbits covering densely invariant manifolds of codimension 3 .

The paper is organized as follows: in Section 2 we give the precise definition of partially hyperbolic tori which we will use. Lemma 2.1 provides a useful set of canonical coordinates near the torus. Then results on invariant manifolds and a lambda lemma are reviewed. Section 3 is devoted to the main result on the existence of orbits whose closure is very big. Finally two examples are presented in Section 4.

\section{Partially hyperbolic tori and related results}

Let $(\mathcal{X}, \Omega)$ be a $2 n$-dimensional symplectic manifold, that is, $\mathcal{X}$ is a $2 n$-manifold and $\Omega$ is a closed non degenerate 2 form on $\mathcal{X}$. Let $H: \mathcal{X} \rightarrow \mathbb{R}$ be a $C^{r+1}$ Hamiltonian, $r \geq 2$, and let $\Phi_{t}$ be the flow generated by its corresponding vector field, $v_{H}$. We will denote $B_{\delta}^{k}$ the open ball around 0 of radius $\delta$ in $\mathbb{R}^{k}$.

We first review some definitions.

A set $\mathcal{T} \subset \mathcal{X}$ is a $m$-invariant Liouville torus with frequency vector $\omega \in \mathbb{R}^{m}$ if there exists an embedding $i: \mathbb{T}^{m} \longrightarrow \mathcal{X}, \mathcal{T}=i\left(\mathbb{T}^{m}\right), D i \cdot \omega=v_{H} \circ i$. This condition is equivalent to $\Phi_{t} \circ i(\cdot)=i(\cdot+t \omega)$.

$\mathcal{T}$ is called non-resonant if the frequency vector $\omega$ is non-resonant:

$$
\langle k, \omega\rangle \neq 0, \quad \text { for all } k \in \mathbb{Z}^{m} \backslash\{0\}
$$

We remark that if $\mathcal{T}$ is non-resonant, then $\mathcal{T}$ is isotropic (i.e., $i^{*} \Omega=0$, the symplectic two form vanishes on $\mathcal{T}$ ) [14].

An isotropic Liouville invariant torus $\mathcal{T}$ is partially hyperbolic if $m<n$ and there exist two smooth $(n-m)$-dimensional subbundles $E^{u, s}$ of the bundle $T_{\mathcal{T}} \mathcal{X}$, invariant by $\Phi_{t}$, such that the linearized flow is expanding on $E^{u}$ and contracting on $E^{s}$, that 
is, given a Riemannian metric on $\mathcal{X}$, there exists $\sigma>0$ and $c>0$ such that, $\forall z \in \mathcal{T}$, $\forall t \geq 0$,

$$
\begin{aligned}
\left\|D \Phi_{t \mid z} v\right\| & \leq c e^{-\sigma t}\|v\|, & & \forall v \in E_{z}^{s}, \\
\left\|D \Phi_{-t \mid z} v\right\| & \leq c e^{-\sigma t}\|v\|, & & \forall v \in E_{z}^{u} .
\end{aligned}
$$

(See also [3].) We will assume that the bundles $E^{u, s}$ are trivial. If not, they can be trivialized by considering a finite covering of a tubular neighborhood of the torus.

Lemma 2.1. Under the previous conditions, if $\mathcal{T}$ is a partially hyperbolic torus, there exist a neighborhood $U$ of $\mathbb{T}^{m}$ in $\mathbb{R}^{n-m} \times \mathbb{R}^{n-m} \times \mathbb{T}^{m} \times \mathbb{R}^{m}$ and a symplectic diffeomorphism $g: U \rightarrow \mathcal{X}$ such that $g\left(\mathbb{T}^{n}\right)=\mathcal{T}, g^{*} \Omega=d x \wedge d y+d \theta \wedge d I$ and, for any given value of $t, g$ transforms the time $t$ map $\Phi_{t}$ of $H$ into

$$
F(x, y, \theta, I)=\left(A_{+}(\theta) x, A_{-}(\theta) y, \theta+\omega(I)+C(\theta) I, I\right)+f(x, y, \theta, I),
$$

with $\left\|A_{+}^{-1}(\theta)\right\|,\left\|A_{-}(\theta)\right\|<c e^{-\sigma t}, \sigma>0$, and $f=\mathcal{O}\left(\|(x, y, I)\|^{2}\right)$.

The proof is based on symplectic geometry arguments and it is deferred to the Appendix A.

Remark 2.2. Note that Lemma 2.1 implies the existence of a trivial tubular neighborhood of $\mathcal{T}$, that is, a tubular neighborhood diffeomorphic to $\mathbb{R}^{2 n-m} \times \mathbb{T}^{m}$. The key points are that the torus is isotropic and that the bundles $E^{u}$ and $E^{s}$ are trivial. The associated dynamics, as it is shown in the proof, implies that $E^{u} \oplus E^{s}$ is a symplectic bundle. The tubular neighborhood construction gives the existence of a neighborhood $U$ of $\mathcal{T}$ in $\mathcal{X}$ such that $U$ is diffeomorphic to $E^{u} \oplus E^{s} \oplus T^{*} \mathcal{T}$, while Weinstein's Theorem ensures that this diffeomorphism can be chosen to be symplectic. Finally since the cotangent bundle of $\mathcal{T}$ is trivial, the tubular neighborhood is trivial.

Partially hyperbolic tori have invariant stable and unstable manifolds associated to their contracting and expanding bundles. For analytic Hamiltonian systems this result goes back to Graff [13] and Zenhder [18]. Below we quote a more general result applicable to families of differentiable maps, not necessarily symplectic (see [12], also $[9,10])$.

Theorem 2.3. Let $F_{\nu}: B_{\delta}^{p} \times B_{\delta}^{q} \times \mathbb{T}^{k} \times B_{\delta}^{l} \rightarrow \mathbb{R}^{p} \times \mathbb{R}^{q} \times \mathbb{T}^{k} \times \mathbb{R}^{l}, \nu \in B_{\mu}^{i}$ be a family of $C^{r}$ diffeomorphisms, $r \in \mathbb{N} \cup\{\infty\}$, of the form

$$
F_{\nu}(x, y, \theta, I)=\left(A_{\nu+}(\theta) x, A_{\nu-}(\theta) y, \theta+\omega_{\nu}(x, y, I), B_{\nu}(\theta) I\right)+f_{\nu}(x, y, \theta, I) .
$$

If there exist $\lambda>1, \beta \geq 1, K_{\theta} \geq 0$ such that $\lambda^{-1} \beta\left(1+K_{\theta}\right)^{r}<1$, a continuous non-negative function $L(\delta, \mu)$ such that $L(0,0)=0$ and 
1. the functions $A_{\nu \pm}, B_{\nu}, \omega_{\nu}$ and $f_{\nu}$ are $C^{r}$ with respect to $(x, y, \theta, I, \nu)$,

2. $\left.\| A_{\nu_{-}} \theta\right)\left\|<\lambda^{-1},\right\| A_{\nu+}(\theta)\left\|<\lambda^{-1},\right\| B_{\nu}(\theta) \|<\beta, \forall \theta \in \mathbb{T}^{k}$,

3. the function $f_{\nu}=\left(f_{1}, f_{2}, f_{3}, f_{4}\right)$ verifies that

$$
f_{j}(x, y, \theta, I)=\left\langle\varphi_{\nu}(x, y, \theta, I),(x, y, I)\right\rangle, \quad j \neq 3
$$

with $\varphi_{\nu}$ of class $C^{r}$ with respect to $\theta$ and $\nu$ and $\left\|D f_{\nu}(w, \nu)\right\| \leq L(\delta, \mu)$ for $w \in B_{\delta}^{p} \times B_{\delta}^{q} \times \mathbb{T}^{k} \times B_{\delta}^{l}, \nu \in \times B_{\mu}^{i}$,

4. $\left\|\partial_{\theta} f_{3}(w, \nu)\right\| \leq K_{\theta}$ for $w \in B_{\delta}^{p} \times B_{\delta}^{q} \times \mathbb{T}^{k} \times B_{\delta}^{l}, \nu \in \times B_{\mu}^{i}$,

then there exist $\delta_{0}$ and $\mu_{0}$ such that if $0<\delta<\delta_{0}$ and $\nu \in B_{\mu_{0}}^{i}$, the invariant torus $\{x=0, y=0, I=0\}$ of the map $F_{\nu}$ has invariant stable and unstable manifolds which can be represented as the graph of $C^{r}$ functions $\gamma_{\nu}^{s}(y, \theta)$ and $\gamma_{\nu}^{u}(x, \theta)$ respectively, verifying $\gamma_{\nu}^{s, u}(0, \theta)=0$. The forward iterates of the points on the stable manifold tend to the torus, exponentially fast, as well as the backward iterates of points on the unstable manifold.

Moreover, these functions $\gamma^{s, u}$ are unique functions defined on $B_{\delta}^{p, q} \times \mathbb{T}^{k}$ in the space

$$
\Sigma_{c, M}=\left\{\gamma \text { Lipschitz, lip } \gamma \leq c,\|\gamma(z, \theta)\| \leq M\|z\|\left\|\theta-\theta^{\prime}\right\|\right\}
$$

with $c=\mathcal{O}\left(L^{-1}\right)$ and $M=\mathcal{O}\left(\delta^{-1}\right)$, such that their graphs are invariant by $F_{\nu}$.

Remark 2.4. In fact, Theorem 2.3 is proved in [12] under more general conditions. In particular, in the $C^{k}$ case, $k<\infty$, the dynamics on the torus does not need to be a rotation, which in the present formulation is implicit in hypothesis 3 .

Remark 2.5. Although Theorem 2.3 applies to maps, the fact that the invariant stable and unstable manifolds are unique implies that the same assertion holds true for the flow $\Phi_{t}$ of a differential equation, provided that, for each $t>0, \Phi_{t}$ satisfies the hypotheses of Theorem 2.3, with $\lambda=e^{\sigma t}, \beta=e^{\rho t}$, for some $\sigma>\rho$.

From Theorem 2.3 it follows that a partially hyperbolic isotropic torus, as defined before, has stable and unstable manifolds. Next proposition deals with tori close to a partially hyperbolic isotropic trivial torus.

Proposition 2.6. Let $\mathcal{T}$ be a partially hyperbolic invariant isotropic trivial torus, as defined before. If $\mathcal{T}^{\prime}$ is a Liouville invariant torus $C^{1}$ close enough to $\mathcal{T}$, then $\mathcal{T}^{\prime}$ is also partially hyperbolic and possesses invariant stable and unstable manifolds, which are $C^{1}$ close to those of $\mathcal{T}$. 
Proof. In the variables given by Lemma $2.1,(x, y, \theta, I)$, the torus $\mathcal{T}$ is parameterized by $(0,0, \theta, 0)$, with $\theta \in \mathbb{T}^{m}$. If $\mathcal{T}^{\prime}$ is $C^{1}$ close enough to $\mathcal{T}$, it can be parameterized by $(X(\theta), Y(\theta), \theta, R(\theta))$, where the functions $X, Y, R$ have derivatives close to 0 .

It is clear that the relations

$$
\tilde{x}=x-X(\theta), \quad \tilde{y}=y-Y(\theta), \quad \tilde{\theta}=\theta, \quad \tilde{I}=I-R(\theta),
$$

define a close to the identity change of variables, $g: V \rightarrow U, U$ being a neighborhood of $\mathcal{T}^{\prime}$ and $V$ a neighborhood of $\{\tilde{x}=0, \tilde{y}=0, \tilde{I}=0\}$. In these new variables $\mathcal{T}^{\prime}$ is given by $\{\tilde{x}=0, \tilde{y}=0, \tilde{I}=0\}$. Furthermore, the flow $\tilde{\Phi}_{t}=g^{-1} \circ \Phi_{t} \circ g$ is

$$
\tilde{\Phi}_{t}(\tilde{x}, \tilde{y}, \tilde{\theta}, \tilde{I})=\left(A_{+}(\tilde{\theta}) \tilde{x}, A_{-}(\tilde{\theta}) \tilde{y}, \tilde{\theta}+\omega(\tilde{I})+C(\tilde{\theta})(\tilde{I}+R(\tilde{\theta})), \tilde{I}\right)+\tilde{f}(\tilde{x}, \tilde{y}, \tilde{\theta}, \tilde{I}),
$$

where the functions $A_{+}, A_{-}, \omega$ are those of $(2.2)$ and $\tilde{f}=\left(\tilde{f}_{1}, \tilde{f}_{2}, \tilde{f}_{3}, \tilde{f}_{4}\right)$. Note that, since $\mathcal{T}^{\prime}$ is invariant and is given by $\{\tilde{x}=0, \tilde{y}=0, \tilde{I}=0\}$, we have that $\tilde{f}_{j \mid \mathcal{T}^{\prime}}=0$ for $j \neq 3$ and $\tilde{f}_{3}$ is small. Now $\tilde{f}$ can have linear terms, which are of order $(X, Y, R)$. In these variables, the dynamics on $\mathcal{T}^{\prime}$ is given by $\theta \mapsto \theta+\omega(0)+g(\theta)$, where

$$
g(\theta)=\omega(R(\tilde{\theta}))-\omega(0)+C(\tilde{\theta}) R(\tilde{\theta})+f_{3}(X(\tilde{\theta}), Y(\tilde{\theta}), \tilde{\theta}, R(\tilde{\theta})) .
$$

Since $D g$ is small, we have that $\tilde{\Phi}_{t}$ still satisfies the hypotheses of Theorem 2.3 .

Remark 2.7. We are considering the map (2.2) for a fixed value of $t$. The frequency vector on the torus $\mathcal{T}=\{x=0, y=0, I=0\}$ for the map $\Phi_{t}$ is $\omega_{t}=\omega t$, where $\omega$ is the frequency vector on the torus for the vector field. Note that the non-resonance condition for $\omega_{t}$ is

$$
\left\langle k, \omega_{t}\right\rangle \neq l, \quad \forall k \in \mathbb{Z}^{m} \backslash\{0\}, l \in \mathbb{Z} .
$$

There are values of $t$ such that, although the flow is dense on $\mathcal{T}$, the map $\Phi_{t}$ is not ergodic on $\mathcal{T}$. Nevertheless, it is clear that if $\omega$ is non-resonant, then $\omega_{t}$ is non-resonant, in the map sense, for a dense set of values of $t$.

For partially hyperbolic tori we have the following result [12]

Theorem 2.8 (Lambda lemma). Let $F_{\nu}$ be a map of the form (2.3) satisfying hypotheses 1 to 4 of Theorem 2.3 for $j \geq 2$ and such that the invariant torus $\{x=$ $0, y=0, I=0\}$ bears a non-resonant rotation. Let $W^{s, u}$ be its stable and unstable manifolds. Let $\Gamma$ be an $(p+l)$-dimensional $C^{1}$ manifold intersecting transversely $W^{s}$ at $q_{0}$. Then $W^{u} \subset \overline{\cup_{n>0} F^{n}(\Gamma)}$. Moreover, there exist q-dimensional submanifolds $D$ of $\Gamma$ such that if $D_{n}$ is the connected component of $F^{n}(D) \cap B(0, \delta)$ which contains $F^{n}\left(q_{0}\right)$, for any $\varepsilon>0$ there exist $n_{0}$ such that $T D_{n}$ is $\varepsilon$-close to (a subset of) $T W^{u}$ if $n>n_{0}$. 
We remark that in the case of flows, the invariant manifolds of the time $t$ map never intersect transversely, since this intersection contains at least one orbit. This problem can be overcome by using suitable Poincaré sections. We use a different approach.

Since the invariant manifolds of the flow $\Phi_{t}$ given by Theorem 2.3 are unique and the Hamiltonian $H$ is autonomous, the invariant manifolds do not depend on $t$. Moreover, in the Hamiltonian case, the lambda lemma, as it is presented here, also yields the same conclusion if the intersection of the manifolds is transversal in the energy level (see [11]). Therefore, if the flow verifies that the rotation on the torus is non-resonant, it is possible to choose $t$ such that the rotation for the map $\Phi_{t}$ is also non-resonant. Hence, the lambda lemma applies and we obtain that any manifold intersecting transversely the stable manifold of the torus covers densely the unstable one.

\section{The main result}

Now we present our main

Theorem 3.1. Let $\mathcal{B}$ be an open ball of $\mathbb{R}^{n}, n \geq 3$, and $H: \mathbb{T}^{n} \times \mathcal{B} \longrightarrow \mathbb{R}$ be a $C^{4}$ Hamiltonian. Let $E \in \mathbb{R}$. Suppose that $\mathcal{M}$ is a connected subset of $\{z \in$ $\left.\mathbb{T}^{n} \times \mathbb{R}^{n} ; H(z)=E\right\}$ such that

(1) for each $z=(\theta, I) \in \mathcal{M}$ there is an $(n-k)$-partially hyperbolic invariant torus $\mathcal{T}_{z}$ of $H, k \geq 1$, such that the dynamics on $\mathcal{T}_{z}$ is conjugated to a rotation of frequency $\omega_{z}$,

(D) there exists a subset $\mathcal{C} \subset \mathcal{M}$, countable and dense in $\mathcal{M}$, such that for all $z \in \mathcal{C}, \omega_{z}$ is non-resonant,

(3) there exists $\mathcal{M}_{0} \subset \mathcal{M}$ such that $\mathcal{M}-\mathcal{M}_{0}$ is connected, $\sigma$-compact and dense in $\mathcal{M}$ and for all $z \in \mathcal{M}-\mathcal{M}_{0}$ the stable and unstable manifolds of $\mathcal{T}_{z}$ intersect transversely in the energy level $H(z)=E$.

Then there exists an orbit $\{z(t)\}$ of the system $H$ which is dense in $\mathcal{M}$, that is, $\mathcal{M} \subset \overline{\{z(t) ; t \geq 0\}}$.

Remark 3.2. In the next section we will present an example in which $\mathcal{M}$ is compact and $\mathcal{M}_{0}=\emptyset$.

Remark 3.3. The tori such that $\omega_{z}$ is non resonant are isotropic. By Lemma 2.1 and Theorem 2.3 they have invariant manifolds. By Proposition 2.6 tori which are $C^{1}$ close enough also have invariant manifolds. 
Remark 3.4. We note that the points in $\mathcal{M}$ are on an invariant torus of the system and then its complete orbit is confined inside an invariant torus. We can say that there is no transport in the sense that given two points in different tori there are open neighborhoods (in $\mathcal{M}$ ) of these points such that orbits with initial conditions in the first neighborhood do not pass through the second one. Theorem 3.1 states that there exists an orbit intersecting any neighborhood of any point in $\mathcal{M}$.

Proof. The first step of the proof consists on constructing a sequence of points of $\mathcal{C},\left(z_{j}\right)_{j \geq 1}$, so that the unstable manifold of the $\mathcal{T}_{z_{j}}$ intersects tranversally (in the energy level) the stable manifold of $\mathcal{T}_{z_{j+1}}$, and $\left\{z_{j} ; j \geq 1\right\}$ is dense in $\mathcal{M}$.

For that let $K_{m}$ be a sequence of compact and connex sets of $\mathcal{M}-\mathcal{M}_{0}$ such that

$$
K_{m} \subset K_{m+1}, \quad \bigcup_{m \geq 1} K_{m}=\mathcal{M}-\mathcal{M}_{0}
$$

For each $m$ there exists $\delta_{m}>0$ such that

$$
z, z^{\prime} \in K_{m} \quad \text { and } \quad\left\|z-z^{\prime}\right\|<\delta_{m} \quad \text { implies } \quad W^{u} \mathcal{T}_{z} \pitchfork W^{s} \mathcal{T}_{z^{\prime}}
$$

This property holds because, since the invariant manifolds depend on a $C^{1}$ way with respect to the torus, to be transversal is an open property.

We take

$$
\varepsilon_{m}=\min \left(\delta_{m}, 1 / m\right)
$$

and a finite covering $\left\{B\left(u_{m}^{j}, \varepsilon_{m}\right)\right\}_{1 \leq j \leq j_{m}}$ of $K_{m}$ with balls of radius $\varepsilon_{m}$, centered at points $u_{m}^{j} \in K_{m}$.

Since $K_{m}$ is connected and the covering is finite we can visit all balls of the covering passing from one ball to a neighbor one with non void intersection with it. The important point is that we can pass through each ball at least once, but we do not exclude the possibility of having to pass through some of them several times.

Then taking points in the intersections of the balls and also belonging to $\mathcal{C}$ we find a finite sequence $\left\{v_{m}^{1}, \ldots, v_{m}^{i_{m}}\right\}$ of points such that $W^{u} \mathcal{T}_{v_{m}^{i}} \pitchfork W^{s} \mathcal{T}_{v_{m}^{i+1}}$. We select the points so that the first one in the finite sequence at the level $m$ is in the last ball of the sequence corresponding to the level $m-1$. In particular $\left\|v_{m}^{1}-v_{m-1}^{i_{m-1}}\right\|<\delta_{m-1}$.

In this way, joining these finite sequences we obtain the desired (infinite) sequence $\left(z_{j}\right)_{j \geq 1}$.

The second step consists on looking for the initial condition for the orbit. We will find them in the intersection of a sequence of non empty nested compact sets, suitably choosen by using the lambda lemma. 


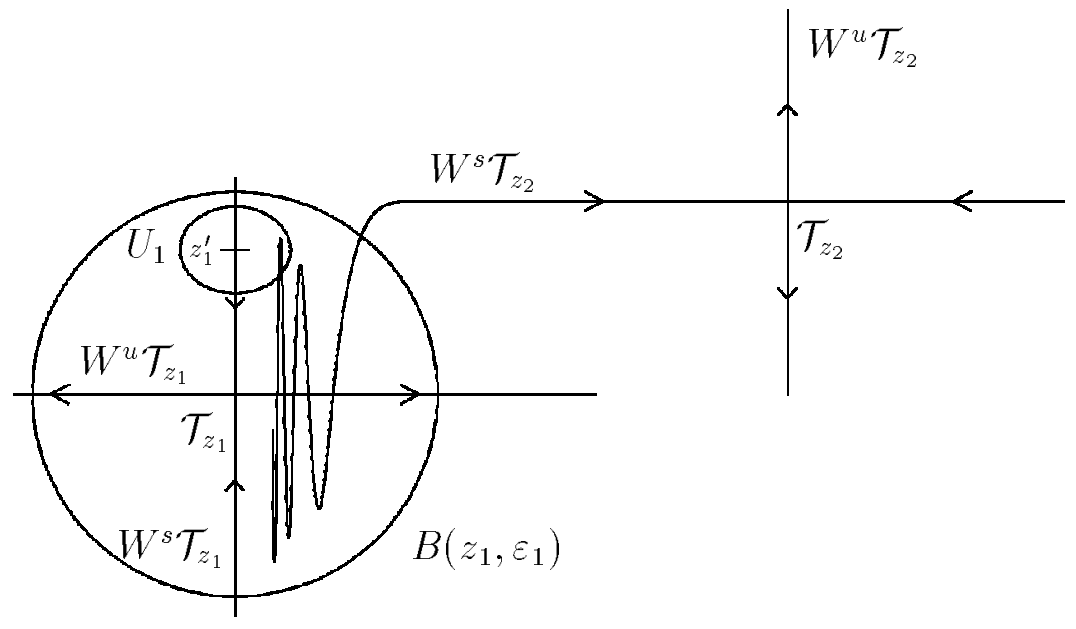

Figure 1: The transition chain. We have identified the points $z_{i}$ with the tori $\mathcal{T}_{z_{i}}$.

In the following $\varepsilon_{l}$ will mean the numbers given by (3.1).

Since the intersection between $W^{u} \mathcal{T}_{z_{j}}$ and $W^{s} \mathcal{T}_{z_{j+1}}$ is transversal and the tori are non-resonant, we can apply the Inclination Lemma to the time reversed flow and we obtain

$$
W^{s} \mathcal{T}_{z_{j}} \subset \overline{W^{s} \mathcal{T}_{z_{j+1}}}, \quad \text { for all } j \geq 1
$$

Also, applying (3.2) inductively, we have that for all $j>1, W^{s} \mathcal{T}_{z_{1}} \subset \overline{W^{s} \mathcal{T}_{z_{j}}}$.

Let $z_{1}^{\prime} \in B\left(z_{1}, \varepsilon_{1}\right) \cap W^{s} \mathcal{T}_{z_{1}}$ and $U_{1} \subset B\left(z_{1}, \varepsilon_{1}\right)$ be an open neighborhood of $z_{1}^{\prime}$ (see Fig. 1). By (3.2), there exist $z_{2}^{\prime} \in B\left(z_{2}, \varepsilon_{2}\right) \cap W^{s} \mathcal{T}_{z_{2}}$, and $t_{1}>0$ such that

$$
\Phi_{-t_{1}}\left(z_{2}^{\prime}\right) \in U_{1} \text {. }
$$

Let $V_{2} \subset B\left(z_{2}, \varepsilon_{2}\right)$ be an open neighborhood of $z_{2}^{\prime}$ and $\tilde{U}_{2}=U_{1} \cap \Phi_{-t_{1}}\left(V_{2}\right)$. We take $U_{2}$ slightly smaller so that $\overline{U_{2}} \subset \tilde{U}_{2}$ and that that $\Phi_{t_{1}}\left(U_{2}\right)$ is an open neighborhood of $z_{2}^{\prime}$.

By induction, let $z_{k}^{\prime} \in B\left(z_{k}, \varepsilon_{k}\right) \cap W^{s} \mathcal{T}_{z_{k}}, U_{k} \subset U_{k-1} \subset \cdots \subset U_{1}$ open sets and $t_{k-1}>0$ such that $\Phi_{t_{1}+\cdots+t_{k-1}}\left(U_{k}\right)$ is a neighborhood of $z_{k}^{\prime}$. By (3.2), there exists $z_{k+1}^{\prime} \in B\left(z_{k+1}, \varepsilon_{k+1}\right) \cap W^{s} \mathcal{T}_{z_{k+1}}$ and $t_{k}>0$ such that

$$
\Phi_{-t_{k}}\left(z_{k+1}^{\prime}\right) \in \Phi_{t_{1}+\cdots+t_{k-1}}\left(U_{k}\right) .
$$

Then, given $V_{k+1} \subset B\left(z_{k+1}, \varepsilon_{k+1}\right)$, a neighborhood of $z_{k+1}^{\prime}$, we define $\tilde{U}_{k+1}=$ $\Phi_{-t_{1}-\cdots-t_{k}}\left(V_{k+1}\right) \cap U_{k}$. We take $U_{k+1}$ slightly smaller so that $\overline{U_{k+1}} \subset \tilde{U}_{k+1}$ and that that $\Phi_{t_{1}+\cdots+t_{k}}\left(U_{k}\right)$ is an open neighborhood of $z_{k+1}^{\prime}$. 
In this way, we have constructed a sequence of open sets, $U_{j}$, such that $U_{j} \subset U_{j-1} \subset$ $\cdots \subset U_{1}$ and $\Phi_{t_{1}+\cdots+t_{j-1}}\left(U_{j}\right) \subset B\left(z_{j}, \varepsilon_{j}\right)$. We have that $\cap_{j \geq 1} U_{j} \supset \cap_{j \geq 1} \overline{U_{j+1}} \neq \emptyset$. The points in $\cap_{j \geq 1} U_{j}$ are initial conditions of orbits dense in $\mathcal{M}$.

\section{Examples}

\subsection{Example 1}

In this subsection we provide an example which satisfies the hypotheses of Theorem 3.1. In fact we give a two parameter family of Hamiltonians such that for an open set of parameters the corresponding Hamiltonian satisfy the hypotheses. The example is the $n$ degrees of freedom family of Hamiltonians

$$
H_{\delta \mu}(\theta, I)=\frac{1}{2}\langle I, I\rangle+\delta\left(\cos \left(2 \pi \theta_{1}\right)-1\right)+\mu I_{1}^{2} \sum_{j=2}^{n} \cos \left(2 \pi \theta_{j}\right)
$$

For such example we will consider the set

$$
\mathcal{M}=\left\{z=(\theta, I) \in \mathbb{T}^{n} \times \mathbb{R}^{n} ; \sum_{j=2}^{n} I_{j}^{2}=E, I_{1}=0, \theta_{1}=0\right\}
$$

with $E>0$ fixed. $\mathcal{M}$ is a compact and connex $2 n-3$ invariant submanifold of the enery level $\left\{H_{\delta \mu}=E\right\}$.

From the observation of the Hamiltonian equations

$$
\begin{aligned}
\dot{\theta}_{1} & =\frac{\partial H}{\partial I_{1}}=I_{1}+2 \mu I_{1} \sum_{j=2}^{n} \cos \left(2 \pi \theta_{j}\right) \\
\dot{\theta}_{j} & =\frac{\partial H}{\partial I_{j}}=I_{j} \\
\dot{I}_{1} & =-\frac{\partial H}{\partial \theta_{1}}=2 \pi \delta \sin \left(2 \pi \theta_{1}\right) \\
\dot{I}_{j} & =-\frac{\partial H}{\partial \theta_{1}}=2 \pi \mu I_{1}^{2} \sin \left(2 \pi \theta_{j}\right)
\end{aligned}
$$

it follows immediately that the set $\mathcal{M}$ is foliated by $n-1$ tori which can be labeled by $n-1$ numbers $\left(\omega_{2}, \ldots, \omega_{n}\right)$. We will write $\omega=\left(\omega_{2}, \ldots, \omega_{n}\right) \in \mathbb{R}^{n-1}$ and then we denote

$$
\mathcal{T}_{\omega}=\left\{z ; \theta_{1}=0, I_{1}=0, I_{2}=\omega_{2}, \ldots, I_{n}=\omega_{n}\right\}
$$


$\mathcal{T}_{\omega}$ are invariant tori of $H_{\delta \mu}$ with frequency vector $\omega$ such that $\frac{1}{2}\langle\omega, \omega\rangle=E$.

Clearly $\mathcal{T}_{\omega} \subset \mathcal{M}$ and $\mathcal{M}=\cup_{\frac{1}{2}\langle\omega, \omega\rangle=E} \mathcal{T}_{\omega}$.

Theorem 3.1 will imply that there is an orbit such that its closure contains $\mathcal{M}$. More precisely

Proposition 4.1. There exists an open set of parameters $\mathcal{P} \subset \mathbb{R}^{2}$ such that for $(\delta, \mu) \in \mathcal{P}, H_{\delta \mu}(\theta, I)$ defined in (4.1) has an orbit $z(t)$ such that $\mathcal{M} \subset \overline{\{z(t) ; t \geq 0\}}$.

Proof. Let us first consider consider the intermediate integrable Hamiltonian

$$
H_{\delta}(\theta, I)=\frac{1}{2}\langle I, I\rangle+\delta\left(\cos \left(2 \pi \theta_{1}\right)-1\right) .
$$

The tori $\mathcal{T}_{\omega}$ introduced in (4.2) are also invariant by $H_{\delta}$. They are partially hyperbolic tori and have one dimensional stable and unstable directions.

We can represent the stable and unstable manifolds of the tori $\mathcal{T}_{\omega}, W_{\delta}^{s}, W_{\delta}^{u}$, as graphs of explicit functions. To get these functions we use that they have to be in the energy level $H_{\delta}(z)=E$ so that

$$
\frac{1}{2} I_{1}^{2}+\frac{1}{2} \sum_{j=2}^{n} I_{j}^{2}+\delta\left(\cos \left(2 \pi \theta_{1}\right)-1\right)=E=\frac{1}{2} \sum_{j=2}^{n} \omega_{j}^{2} .
$$

Then

$$
I_{1}= \pm 2 \sqrt{\delta} \sin \pi \theta_{1}, \quad\left(I_{2}, \ldots, I_{n}\right)=\omega .
$$

We see that the stable and unstable manifolds of $\mathcal{T}$ coincide. Then every orbit in it is a homoclinic orbit.

We consider points in the invariant manifold with $\theta_{1}=1 / 2$, that is,

$$
\left(1 / 2, \theta_{2}^{0}, \ldots, \theta_{n}^{0}, \sqrt{2 \delta}, \omega_{2}, \ldots, \omega_{n}\right)
$$

The homoclinic orbit $(\theta(t), I(t))$ such that at $t=t_{0}$, passes through that point is

$$
\begin{aligned}
\theta_{1}(t) & =\frac{2}{\pi} \arctan \left(e^{2 \pi \sqrt{\delta}\left(t-t_{0}\right)}\right) & I_{1}(t) & =\frac{2 \sqrt{\delta}}{\cosh \left(2 \pi \sqrt{\delta}\left(t-t_{0}\right)\right)}, \\
\theta_{2}(t) & =\theta_{2}^{0}+\omega_{2}\left(t-t_{0}\right), & I_{2}(t) & =\omega_{2}, \\
& \vdots & \vdots & \\
\theta_{n}(t) & =\theta_{n}^{0}+\omega_{n}\left(t-t_{0}\right), & I_{n}(t) & =\omega_{n} .
\end{aligned}
$$


We consider our Hamiltonian $H_{\delta \mu}$ as a perturbation of $H_{\delta}$ and then we can study the invariant manifolds of $H_{\delta \mu}$ by perturbative techniques from the invariant manifolds of $H_{\delta}$.

Since $H_{\delta \mu}$ satisfies the reversibility condition

$$
H_{\delta \mu}(\theta, I)=H_{\delta \mu}\left(1-\theta_{1},-\theta_{2}, \ldots,-\theta_{n}, I\right) .
$$

we can argue easily that the stable and unstable manifolds of $\mathcal{T}_{\omega}$ actually intersect. Indeed (4.4) implies that if $z(t)=\left(\theta_{1}(t), \theta_{2}(t), \ldots, \theta_{n}(t), I(t)\right)$ is a solution then $\tilde{z}(t)=\left(1-\theta_{1}(-t),-\theta_{2}(-t), \ldots,-\theta_{n}(-t), I(-t)\right)$ is also a solution. Then if $z(t)$ is a solution with initial condition on $W^{s} \cap P$ with $P=\left\{\theta_{1}=1 / 2, \theta_{2}=0, \ldots, \theta_{n}=0\right\}$ at $t=0$, that is $d\left(z(t), \mathcal{T}_{\omega}\right) \rightarrow 0$ as $t \rightarrow \infty$ then $d\left(z(t), \mathcal{T}_{\omega}\right) \rightarrow 0$ as $t \rightarrow-\infty$. Note that the points $(1,0, \ldots, 0, \omega)$ are identified with $(0,0, \ldots, 0, \omega)$. Since the manifolds vary smoothly with respect to $\mu$ and since, by (4.3), when $\mu=0$ the stable manifold intersects transversaly $P$, we have that they also intersect for $\mu \neq 0$ small. The points of intersection are initial conditions of homoclinic orbits.

To study the tranvesality of the intersection we use the Poincaré-Melnikov method (see, for instance, [11]). Following that reference Let $M=\left(M_{2}, \ldots, M_{n}\right)$ be Melnikov vector with

$$
M_{j}=M_{j}\left(t_{0}, \theta_{2}^{0}, \ldots, \theta_{n}^{0}\right)=-\int_{-\infty}^{\infty} D_{\theta_{j}^{0}} K(\theta(s), I(s)) d s, \quad j=2, \ldots, n,
$$

where $K=\mu I_{1}^{2} \sum_{j=2}^{n} \cos \left(2 \pi \theta_{j}\right)$ and $(\theta(s), I(s))$ is a homoclinic orbit of the Hamiltonian $H_{\delta}$ with $\theta_{j}\left(t_{0}\right)=\theta_{j}^{0}$. For this example

$$
M_{j}=\int_{-\infty}^{\infty} \frac{8 \pi \delta}{\cosh ^{2}\left(2 \pi \sqrt{\delta}\left(s-t_{0}\right)\right)} \sin \left(2 \pi \theta_{j}^{0}+2 \pi \omega_{j}\left(s-t_{0}\right)\right) d s .
$$

If det $D_{\left(\theta_{2}^{0}, \ldots, \theta_{n}^{\circ}\right)} M(0,0) \neq 0$ the intersection along the homoclinic orbit is tranversal, for values of $\mu$ small enough,

In our case, $D_{\left(\theta_{2}^{0}, \ldots, \theta_{n}^{0}\right)} M(0,0)=\operatorname{diag}\left(g_{2}, \ldots, g_{n}\right)$, where

$$
g_{j}=(2 \pi)^{2} \int_{-\infty}^{\infty} \frac{4 \delta}{\cosh ^{2}(2 \pi \sqrt{\delta} s)} \cos \left(2 \pi \omega_{j} s\right) d s, \quad j=2, \ldots, n .
$$

A direct computation via residue theory shows that

$$
g_{j}=2 \pi^{2} \frac{4 \omega_{j}}{\sinh \left(\frac{\pi \omega_{j}}{2 \sqrt{\delta}}\right)}, \quad j=2, \ldots, n .
$$

In particular, $g_{j} \neq 0$ for all $\omega_{j} \in \mathbb{R}$. Therefore, since $\mathcal{M}$ is compact, there exists $\mu_{0}>0$ such that, for all $\mu,|\mu|<\mu_{0}$, and for all $z \in \mathcal{M}$, the invariant manifolds 
of $\mathcal{T}_{z}$, for the Hamiltonian $H_{\delta \mu}$, intersect transversaly. Proposition 4.1 follows from the fact that the tori with non-resonant frequencies are dense in $\mathcal{M}$ and Theorem 3.1 .

\subsection{Example 2. Arnol'd example}

Consider the Arnol'd Hamiltonian [1]

$$
H_{\delta \mu}(\theta, I)=\frac{1}{2}\left(I_{1}^{2}+I_{2}^{2}\right)+I_{3}+\delta\left(\cos \left(\theta_{1}\right)-1\right)\left(1+\mu\left(\sin \theta_{2}+\cos \theta_{3}\right)\right) .
$$

In this case, for any $E$, the manifold $\mathcal{M}=\left\{I_{2}^{2}+2 I_{3}=E, I_{1}=0, \theta_{1}=0\right\}$ is 3 dimensional and it is foliated by 2 dimensional tori. Therefore, the tori in $\mathcal{M}$ can be described by a single parameter, which can be $I_{2}$.

In [1] it is proved that given two real numbers $0<a<b$, for any $\delta>0$, there exists $\mu_{0}$ such that for any $0<\mu<\mu_{0}$ the Hamiltonian system (4.5) has an orbit with $I_{2}\left(t_{0}\right)<a, I_{2}\left(t_{1}\right)>b$, for some $t_{0}<t_{1}$. It is clear that $I_{2}$ reaches all values between $a$ and $b$ as $t$ goes from $t_{0}$ to $t_{1}$.

Theorem 3.1 can also be applied to this example in the following way: consider the interval $[a, b]$ and let $\left(J_{k}\right)_{1 \leq k \leq N}$ be a partition of the interval $[a, b]$. Then we have

Proposition 4.2. For any sequence $\left(k_{1}, k_{2}, \ldots\right), k_{i} \in \mathbb{N}, 1 \leq k_{i} \leq N$, there exists an open set of parameters $\mathcal{P} \subset \mathbb{R}^{2}$ such that for $(\delta, \mu) \in \mathcal{P}, H_{\delta \mu}(\theta, I)$ defined in (4.5) has an orbit $z(t)=(\theta(t), I(t))$ verifying that, for an increasing sequence of time $t_{1}<t_{2}<\cdots, I_{2}\left(t_{i}\right) \in J_{k_{i}}$.

Proof. In [1] it is checked that the manifold $\mathcal{M}_{a, b}=\mathcal{M} \cap\left\{a \leq I_{2} \leq b\right\}$ verifies the hypotheses of Theorem 3.1 for an open subset of parameters.

We define $\mathcal{M}_{j}=\left\{z=(\theta, I) \in \mathcal{M}_{a, b}: I_{2} \in J_{j}\right\}$. It is clear that $\mathcal{M}_{a, b}=\cup_{j} \mathcal{M}_{j}$. Since, by Theorem 3.1, there is an orbit $z(t)$ covering densely $\mathcal{M}_{a, b}$, there exists an increasing sequence of times $t_{1}<t_{2}<\cdots$ such that $z\left(t_{i}\right)$ is arbitrarily close to points in $\mathcal{M}_{j}$. In particular, the sequence can be chosen in such a way that $I_{2}\left(t_{i}\right) \in J_{k_{i}}$.

\section{A Proof of Lemma 2.1}

The proof consists on finding a symplectic basis $\left\{u_{1}(z), \ldots, u_{2 n}(z)\right\}$ of $T_{z} \mathcal{X}$ for each $z \in \mathcal{T}$ so that

- $\left\{u_{1}(z), \ldots, u_{n-m}(z)\right\}$ is a basis of $E_{z}^{u}$, 
- $\left\{u_{n-m+1}(z), \ldots, u_{n}(z)\right\}$ is a basis of $T_{z} \mathcal{T}$,

- $\left\{u_{n+1}(z), \ldots, u_{2 n-m}(z)\right\}$ is a basis of $E_{z}^{s}$.

Then we see that in a coordinate system associated to this basis, the map $\Phi_{t}$ has the claimed form (2.2).

The construction of the basis will proceed in several steps.

By taking coordinates on $\mathcal{T}$, we can identify a point $z \in \mathcal{T}$ by $\theta=\left(\theta_{1}, \ldots, \theta_{m}\right)$, $\theta \in \mathbb{T}^{m}$. Hence, the constant vector fields $\left\{\frac{\partial}{\partial \theta_{1}}, \ldots, \frac{\partial}{\partial \theta_{m}}\right\}$ on $\mathcal{T}$, on each $z \in \mathcal{T}$, expand $T_{z} \mathcal{T}$.

The time $t$ map $\Phi_{t}$ is a symplectic map, that is, it verifies that $\Phi_{t}^{*} \Omega=\Omega$.

We write $z_{t}=\Phi_{t} z$ and we represent by $\Omega_{\mid z}$ the restriction of $\Omega$ to $T_{z} \mathcal{X}$.

Taking $v, w \in E^{u}$, since $\Phi_{-t}$ is contracting on $E^{u}$, we find that

$$
\Omega_{\mid z}(v, w)=\lim _{t \rightarrow-\infty} \Omega_{\mid z_{t}}\left(D \Phi_{t}(z) v, D \Phi_{t}(z) w\right)=0 .
$$

This implies that $\Omega_{\mid E^{u}}=0$. In an analogous way one sees that $\Omega_{\mid E^{s}}=0$.

Since $\Phi_{t \mid \mathcal{T}}$ is a rotation, for $z \in \mathcal{T}$ we will simply write that $D \Phi_{t}(z)_{\mid T_{z} \mathcal{T}}=\mathrm{Id}$, although $D \Phi_{t}(z)_{\mid T_{z} \mathcal{T}}: T_{z} \mathcal{T} \longrightarrow T_{z_{t}} \mathcal{T}$. Moreover, since $\mathcal{T}$ is isotropic, invariant, and $D \Phi_{t \mid \mathcal{T}}=$ Id we have that, for all $z \in \mathcal{T}, E_{z}^{u} \oplus T_{z} \mathcal{T}$ is a Lagrangian subspace of $T_{z} \mathcal{X}$. For that it only remains to be checked that if $v \in E_{z}^{u}$ and $w \in T_{z} \mathcal{T}$ then $\Omega_{\mid z}(v, w)=0$. This follows from $\Omega_{\mid z}(v, w)=\lim _{t \rightarrow-\infty} \Omega_{\mid z_{t}}\left(D \Phi_{t}(z) v\right.$, Id $\left.w\right)=0$. In the same way one sees that $E_{z}^{s} \oplus T_{z} \mathcal{T}$ is also Lagrangian.

Since $E^{u}$ is trivial, there exist vector fields on $\mathcal{X}, e_{j}(z), 1 \leq j \leq n-m$, such that, for all $z \in \mathcal{T},\left\{e_{1}(z), \ldots, e_{n-m}(z)\right\}$ is a basis of $E_{z}^{u}$. We define $e_{n-m+j}(z)=\frac{\partial}{\partial \theta_{j}}$, for $1 \leq j \leq m$. Then $\left\{e_{1}(z), \ldots, e_{n}(z)\right\}$ is a basis of $E_{z}^{u} \oplus T_{z} \mathcal{T}$.

We claim that there exist $\left\{e_{n+1}(z), \ldots, e_{2 n}(z)\right\}$, vector fields on $\mathcal{X}$, such that, for all $z \in \mathcal{T},\left\{e_{1}(z), \ldots, e_{2 n}(z)\right\}$ is a canonical basis of $T_{z} \mathcal{X}$, that is

$$
\Omega\left(e_{i}, e_{j}\right)=0, \Omega\left(e_{n+i}, e_{n+j}\right)=0, \Omega\left(e_{i}, e_{n+j}\right)=\delta_{i, j}, \quad i, j=1, \ldots, n,
$$

where the preceding expressions are evaluated at $z \in \mathcal{T}$.

Indeed, let $\left\{\eta_{1}, \ldots, \eta_{n}\right\}$ the dual forms of $\left\{e_{1}, \ldots, e_{n}\right\}$, that is, $\eta_{i}\left(e_{j}\right)=\delta_{i, j}, 1 \leq i, j \leq$ $n$. Let $\Omega^{\sharp}$ denote the diffeomorphism between 1 -forms and vector fields induced by the symplectic form $\Omega$. We define

$$
\tilde{e}_{n+j}=\Omega^{\sharp} \eta_{j}, \quad 1 \leq j \leq n .
$$

Since $\Omega_{\mid E^{u} \oplus T \mathcal{T}}=0$, it follows that

$$
\Omega\left(e_{i}, e_{j}\right)=0, \quad \Omega\left(e_{i}, \tilde{e}_{n+j}\right)=\delta_{i, j}, \quad i, j=1, \ldots, n .
$$


We take $e_{n+i}=\tilde{e}_{n+i}+\sum_{j=1}^{n} d_{j i} e_{j}$. The conditions

$$
\Omega\left(e_{n+i}, e_{n+j}\right)=0, \quad i, j=1, \ldots, n,
$$

are equivalent to

$$
\Omega\left(\tilde{e}_{n+i}, \tilde{e}_{n+j}\right)-d_{i j}+d_{j i}=0, \quad i, j=1, \ldots, n,
$$

which obviously can be solved. Furthermore, the new $e_{n+i}$ satisfy (A.1).

For a point $z \in \mathcal{T}$, we introduce the following subspaces of $T_{z} \mathcal{X}$ :

$$
F_{z}^{3}=\left\langle e_{n+1}(z), \ldots, e_{2 n-m}(z)\right\rangle, \quad F_{z}^{4}=\left\langle e_{2 n-m+1}(z), \ldots, e_{2 n}(z)\right\rangle .
$$

From the invariance of $E^{u}$ we have that, for $i=1, \ldots, n-m, D \Phi_{t}(z) e_{i}(z)=$ $\sum_{j=1}^{n-m} c_{j i} e_{j}\left(z_{t}\right)$, and the matrix $\left(c_{j i}\right)_{1 \leq i, j \leq n-m}$ is non singular. We claim that if $e_{2 n-m+j}(z) \in F_{z}^{4}$ then $D \Phi_{t}(z) e_{2 n-m+j}(z)-e_{2 n-m+j}\left(z_{t}\right) \in E_{z_{t}}^{u} \oplus T_{z_{t}} \mathcal{T}$. Indeed, for $1 \leq j \leq m$ we write

$$
D \Phi_{t}(z) e_{2 n-m+j}(z)=w_{j}+\sum_{k=1}^{n-m} \alpha_{k j} e_{n+k}\left(z_{t}\right)+\sum_{l=1}^{m} \beta_{l j} e_{2 n-m+l}\left(z_{t}\right)
$$

with $w_{j} \in E_{z_{t}}^{u} \oplus T_{z_{t}} \mathcal{T}$. Taking $i$ with $1 \leq i \leq m$, from

$$
\delta_{i j}=\Omega_{\mid z}\left(e_{n-m+i}(z), e_{2 n-m+j}(z)\right)=\Omega_{\mid z_{t}}\left(e_{n-m+i}\left(z_{t}\right), D \Phi_{t}(z) e_{2 n-m+j}(z)\right)
$$

we get $\beta_{i j}=\delta_{i j}$, and taking $i$ with $1 \leq i \leq n-m$, from

$$
\begin{aligned}
0 & =\Omega_{\mid z}\left(e_{i}(z), e_{2 n-m+j}(z)\right)=\Omega_{\mid z_{t}}\left(D \Phi_{t}(z) e_{i}(z), D \Phi_{t}(z) e_{2 n-m+j}(z)\right) \\
& =\Omega_{\mid z_{t}}\left(\sum_{l=1}^{n-m} c_{l i} e_{l}\left(z_{t}\right), w_{j}+\sum_{k=1}^{n-m} \alpha_{k j} e_{n+k}\left(z_{t}\right)+e_{2 n-m+j}\left(z_{t}\right)\right)=\sum_{j=1}^{n-m} c_{l i} \alpha_{l j} .
\end{aligned}
$$

The fact that the matrix $\left(c_{l i}\right)$ is invertible implies $\alpha_{l j}=0$, for all $l, j$.

We claim that $D \Phi_{t}(z) F_{z}^{3} \subset E_{z_{t}}^{u} \oplus T_{z_{t}} \mathcal{T} \oplus F_{z_{t}}^{3}$. Indeed, for $1 \leq j \leq n-m$ we write

$$
D \Phi_{t}(z) e_{n+j}(z)=w_{j}+\sum_{k=1}^{m} \gamma_{k j} e_{2 n-m+k}\left(z_{t}\right), \quad w_{j} \in E_{z_{t}}^{u} \oplus T_{z_{t}} \mathcal{T} \oplus F_{z_{t}}^{3} .
$$

Then, for $1 \leq i \leq m$

$$
\begin{aligned}
0 & =\Omega_{\mid z}\left(e_{n-m+i}(z), e_{n+j}(z)\right)=\Omega_{\mid z_{t}}\left(D \Phi_{t}(z) e_{n-m+i}(z), D \Phi_{t}(z) e_{n+j}(z)\right) \\
& =\Omega_{\mid z_{t}}\left(e_{n-m+i}\left(z_{t}\right), \sum_{k=1}^{m} \gamma_{k j} e_{2 n-m+k}\left(z_{t}\right)\right)=\gamma_{i j}
\end{aligned}
$$


Next we claim that $E_{z}^{s} \subset E_{z}^{u} \oplus T_{z} \mathcal{T} \oplus F_{z}^{3}$.

We write $v \in E_{z}^{s}$ as $v=w+\sum_{j=1}^{m} \alpha_{j} e_{2 n-m+j}(z)$ with $w \in E_{z}^{u} \oplus T_{z} \mathcal{T} \oplus F_{z}^{3}$. Then, for $1 \leq i \leq m$, since for any $v \in E_{z}^{s}, D \Phi_{t}(z) v$ tends to zero (exponentially fast) when $t \rightarrow \infty$,

$$
\begin{aligned}
0 & =\lim _{t \rightarrow \infty} \Omega_{\mid z_{t}}\left(e_{n-m+i}\left(z_{t}\right), D \Phi_{t}(z) v\right)=\Omega_{\mid z}\left(e_{n-m+i}(z), v\right) \\
& =\Omega_{\mid z}\left(e_{n-m+i}(z), w+\sum_{j=1}^{m} \alpha_{j} e_{2 n-m+j}(z)\right)=\alpha_{i}
\end{aligned}
$$

Now we are going to look for a good basis of $E^{s}$. Since $E^{s}$ is trivial there are $n-m$ independent vector fields $\tilde{u}_{j}, n+1 \leq j \leq 2 n-m$ such that $\left\{\tilde{u}_{n+1}(z), \ldots, \tilde{u}_{2 n-m}(z)\right\}$ is a basis of $E_{z}^{s}$ for all $z \in \mathcal{T}$. Since $E^{s} \subset E^{u} \oplus T \mathcal{T} \oplus F^{3}$, then

$$
\tilde{u}_{n+j}=\sum_{l=1}^{n-m} a_{l j} e_{n+l}+\sum_{l=1}^{n} b_{l j} e_{l}, \quad 1 \leq j \leq n-m,
$$

where the $(n-m) \times(n-m)$ matrix $A=\left(a_{l j}\right)$ is nonsingular.

We construct another basis $\left\{u_{n+1}, \ldots, u_{2 n-m}\right\}$ of $E^{s}$ in such a way that

$$
\left\{e_{1}, \ldots, e_{n-m}, u_{n+1}, \ldots, u_{2 n-m}\right\}
$$

is symplectic. Let

$$
u_{n+j}=\sum_{l=1}^{n-m} c_{l j} \tilde{u}_{n+l}, \quad 1 \leq j \leq n-m
$$

We impose that

$$
\begin{array}{cl}
\Omega\left(e_{i}, u_{n+j}\right)=\delta_{i j}, & 1 \leq i \leq n-m \\
\Omega\left(u_{n+i}, u_{n+j}\right)=0, & 1 \leq i \leq n-m .
\end{array}
$$

Condition (A.4) is automatically satisfied because $E^{s}$ is isotropic. From (A.3) we get

$$
\begin{aligned}
\delta_{i j}=\Omega\left(e_{i}, \sum_{l=1}^{n-m} c_{l j} \tilde{u}_{n+l}\right) & =\sum_{l=1}^{n-m} c_{l j} \Omega\left(e_{i}, \sum_{k=1}^{n-m} a_{k l} e_{n+k}+\sum_{k=1}^{n} b_{k l} e_{k}\right) \\
& =\sum_{l=1}^{n-m} c_{l j} \sum_{k=1}^{n-m} a_{k l} \delta_{i k}=\sum_{l=1}^{n-m} c_{l j} a_{i l} .
\end{aligned}
$$

If we denote $A=\left(a_{i j}\right), C=\left(c_{i j}\right)$, the condition (A.3) is equivalent to $A C=\mathrm{Id}$ so that it suffices to take $C=A^{-1}$ to fulfill it. 
Finally we look for vector fields $u_{j}, j=2 n-m+1, \ldots, 2 n$, so that

$$
\left\{e_{1}, \ldots, e_{n}, u_{n+1}, \ldots, u_{2 n-m}, u_{2 n-m+1}, \ldots, u_{2 n}\right\}
$$

is a symplectic basis of $T_{\mid \tau} \mathcal{M}$. This can be accomplished by taking

$$
u_{2 n-m+j}=e_{2 n-m+j}+\sum_{l=1}^{n} d_{l j} e_{l}, \quad 1 \leq j \leq m,
$$

and choosing $d_{l j}$ so that for $1 \leq j \leq m$,

$$
\begin{aligned}
& \Omega\left(e_{i}, u_{2 n-m+j}\right)=0, \quad \text { for } 1 \leq i \leq n-m, \\
& \Omega\left(e_{n-m+i}, u_{2 n-m+j}\right)=\delta_{i j}, \quad \text { for } 1 \leq i \leq m, \\
& \Omega\left(u_{n+i}, u_{2 n-m+j}\right)=0, \quad \text { for } 1 \leq i \leq n-m, \\
& \Omega\left(u_{2 n-m+i}, u_{2 n-m+j}\right)=0, \quad \text { for } 1 \leq i \leq m .
\end{aligned}
$$

The form (A.5) guarantees that (A.6) and (A.7) are satisfied. The conditions (A.8) and (A.9) are equivalent to

$$
\begin{aligned}
& \Omega\left(u_{n+i}, e_{2 n-m+j}\right)+d_{i j}=0 \\
& -d_{n-m+i, j}+d_{n-m+j, i}=0 .
\end{aligned}
$$

Condition (A.10) determines the values of $d_{i j}$ for $1 \leq i \leq n-m, 1 \leq j \leq m$. Condition (A.11) only requires the submatrix $\left(d_{n-m+i, j}\right)_{1 \leq i, j \leq m}$ to be symmetric.

Finally, repeating the same arguments, we have that the image of the vector bundle $\left\langle u_{2 n-m+1}, \ldots, u_{2 n}\right\rangle$ is spanned by $\left\langle e_{n-m+1}, \ldots, e_{n}, u_{2 n-m+1}, \ldots, u_{2 n}\right\rangle$.

Indeed, by (A.2) $D \Phi_{t}(z) e_{2 n-m+j}(z)=w_{j}+\sum_{l=1}^{m} \beta_{l j} e_{2 n-m+l}\left(z_{t}\right), w_{j} \in E_{z_{t}}^{u} \oplus T_{z_{t}} \mathcal{T}$, so that $\Pi^{s} D \Phi_{t}(z) e_{2 n-m+j}(z)=0$. Then, by $\left(\right.$ A.5) we have that $\Pi^{s} D \Phi_{t}(z) u_{2 n-m+j}(z)=$ 0 . Using again (A.2) we obtain that $D \Phi_{t}(z) u_{2 n-m+j}-u_{2 n-m+j} \in T_{z} \mathcal{T}$.

We define $u_{j}=e_{j}$ for $1 \leq j \leq n$. Now, we define coordinates in $\mathcal{X}$ by means of these vector fields and the exponential map induced by the Riemannian metric on $\mathcal{X}$ : the coordinates

$$
\left(x_{1}, \ldots, x_{n-m}, y_{1}, \ldots, y_{n-m}, \theta_{1}, \ldots, \theta_{m}, I_{1}, \ldots, I_{m}\right)
$$

will denote the point

$$
\begin{aligned}
\exp \left(x_{1} u_{1}(z)+\cdots+x_{n-m} u_{n-m}(z)+y_{1} u_{n+1}(z)\right. & +\cdots+y_{n-m} u_{2 n-m}(z) \\
& \left.+I_{1} u_{2 n-m+1}(z)+\cdots+I_{m} u_{2 n}(z)\right)
\end{aligned}
$$

where $z \in \mathcal{T}$ has coordinates $\left(\theta_{1}, \ldots, \theta_{m}\right)$. 
We consider the canonical 2 form $\tilde{\Omega}=d x \wedge d y+d \theta \wedge d I$, which is non-degenerate and closed (actually it is exact). By construction, we have that $\Omega$ and $\tilde{\Omega}$ coincide on $\mathcal{T}$. Therefore, by Weinstein's theorem (see, for instance [15]), there exists a diffeomorphism $g$ from a neighborhood of $\mathcal{T}$ to $\mathbb{R}^{2 n-m} \times \mathbb{T}^{m}$ such that $g_{\mid \mathcal{T}}=\mathrm{Id}$, $g^{*} \Omega=\tilde{\Omega}$ and $D g_{\mid \mathcal{T}}=\mathrm{Id}$. Then, it is clear that $D \Phi_{t}$, evaluated on $z=(0,0, \theta, 0) \in \mathcal{T}$, has the form

$$
\left(\begin{array}{cccc}
A_{+}(\theta) & 0 & 0 & 0 \\
0 & A_{-}(\theta) & 0 & 0 \\
0 & 0 & \operatorname{Id} & C(\theta) \\
0 & 0 & 0 & \operatorname{Id}
\end{array}\right)
$$

where $A_{+}$is the restriction of $D \Phi_{t}$ on $E^{u}$ in this basis and $A_{-}$is the restriction of $D \Phi_{t}$ on $E^{s}$, and Id is the identity matrix. By (2.1), we have that $\left\|A_{+}^{-1}(\theta)\right\|,\left\|A_{-}(\theta)\right\| \leq$ $c e^{-\sigma t}$. The rest of the claim follows from Taylor's theorem.

\section{B Acknowledgements}

E.F acknowledges the partial support of the the Spanish Grant DGICYT, PB940215, the Catalan Grant CIRIT 1998 SGR-00041 and the INTAS project 97-0771. P.M. acknowledges the partial support of the Catalan Grant CIRIT 1998 SGR-00041.

\section{References}

[1] V.I. Arnol'd. Instability of dynamical systems with several degrees of freedom. Soviet Math. Dokl. 5 (1964) 581-585.

[2] V.I. Arnol'd \& A. Avez. Problèmes Ergodiques de la Mécanique Classique, Gauthier-Villars: Paris, 1967.

[3] S. Bolotin \& D. Treschev. Unbounded growth of energy in nonautonomous Hamiltonian systems. Nonlinearity 12 (1999) 365-388.

[4] L. Chierchia \& G. Gallavotti. Drift and diffusion in phase space. Ann. Int. H. Poincaré Phys. Th. 60 (1994) 1-144.

[5] L. Chierchia L \& E. Valdinoci. A note on the construction of Hamiltonian trajectories along heteroclinic chains. Forum Math 12 (2000) 247-255.

[6] J. Cresson. A $\lambda$-lemma for partially hyperbolic tori and the obstruction property. Letters in Math. Phys. 42 (1997) 363-377. 
[7] A. Delshams, R. de la Llave \& T. Martinez-Seara. A geometric approach to the existence of orbits with unbounded energy in generic periodic perturbations by potential of generic geodesic flows on $\mathbb{T}^{2}$. Comm. Math. Phys. 209 (2000) $353-392$.

[8] R. Douady. Stabilité ou instabilité des points fixes elliptiques. Annal. Sci. de l'ENS 21 (1988) 1-46.

[9] N. Fenichel. Asymptotic stability with rate conditions. Indiana Univ. Math. J. 23 (1974) 1109-1137.

[10] N. Fenichel. Asymptotic stability with rate conditions II. Indiana Univ. Math. J. 26 (1977) 81-93.

[11] E. Fontich \& P. Martín. Arnold diffusion in perturbations of analytic integrable Hamiltonian systems. preprint, 1998.

[12] E. Fontich \& P. Martín. Differentiable invariant manifolds of hyperbolic tori and a $C^{1} \lambda$-Lemma. To appear in Nonlinearity, 1999.

[13] S. Graff. On the conservation of hyperbolic invariant tori for hamiltonian systems. J. Differential Equations 15 (1974) 1-69.

[14] M.R. Herman. Inégalités a priori pour des tores langrangiens invariants par des difféomorphismes symplectiques. Publ. Math. I.H.E.S. 70 (1990) 47-101.

[15] P. Libermann \& C.M. Marle. Symlectic Geometry and Analytical Mechanics. D. Reidel Publishing Company: Dordrecht, 1987.

[16] J.P. Marco. Transition le long des chaînes de tores invariants pour les systèmes hamiltoniens analytiques. Annales de l'I.H.P., Physique Théorique 64 (1996) $205-252$.

[17] P. Perfetti. Fixed point theorems in the Arnol'd model about instability of Hamiltonian dynamical systems. Discrete and Continuous Dyn. Systems 4 (1998) 379-391.

[18] E. Zehnder. Generalized implicit function theorems with applications to some small divisor problems, II. Comm. Pure Appl. Math. 29 (1976) 49-111. 